\title{
Direct numerical simulation of an atomizing biodiesel jet: Impact of fuel properties on atomization characteristics
}

\author{
Yue Ling ${ }^{1 *}$, Guillaume Legros ${ }^{2}$, Stéphane Popinet ${ }^{2}$, Stéphane Zaleski \\ ${ }^{1}$ Department of Mechanical Engineering, Baylor University, Texas, USA \\ ${ }^{2}$ Sorbonne Universités, UPMC, Universitris 06, CNRS, UMR 7190, Institut Jean Le Rond \\ d'Alembert, F-75005, Paris, France \\ ${ }^{*}$ Corresponding author: stanley_ling@baylor.edu
}

\begin{abstract}
The utilization of biodiesel is an effective approach to reduce pollution from internal combustion engines and thus has attracted steadily increasing interest in the recent years. As the viscosity of biodiesel is much higher than that of standard diesel, the atomization characteristics of a biodiesel jet can significantly deviate from those of a standard diesel jet under identical injection conditions. Since atomization of the injected fuel has a strong impact on fuel-air mixing and the following combustion processes, it is important to investigate the atomization of biodiesel and in particular to understand how the fuel properties affect the atomization process and the resulting spray characteristics. In the present study, three-dimensional direct numerical simulations are conducted to investigate atomizing biodiesel and diesel jets. The novel adaptive multiphase solver Basilisk is used for simulations. The statistics of droplets formed in the biodiesel jet is compared to the diesel jet under identical injection conditions.
\end{abstract}

\section{Keywords}

Biodiesel, atomization, direct numerical simulation, Volume-of-fluid method

\section{Introduction}

The utilization of biodiesel is an effective approach to reduce pollution from internal combustion engines and thus has attracted ever increasing interest in recent years. As the material properties of biodiesel are different from those of the standard diesel, the atomization characteristics of a biodiesel jet can significantly deviate from those of a standard diesel jet under identical injection conditions. Since atomization of the injected fuel has a strong impact on the fuel-air mixing and following combustion processes, it is important to investigate the atomization of biodiesel and in particular to understand how the fuel properties affect the atomization process and the resulting spray characteristics.

Atomization of a liquid jet is a process of enormous complexity involving a wide range of spatial scales. As a result, numerical simulation of atomization is very challenging. In the present work, direct numerical simulations of atomizing jets are performed with a novel adaptive multiphase solver, Basilisk [1]. The solver uses the Volume-Of-Fluid (VOF) method to capture the liquid-gas interface. A balanced-force discretization is employed for surface-tension calculation [2]. An octree spatial discretization is utilized, which gives a very important flexibility allowing dynamic grid refinement into user-defined regions. Finally, massively parallel simulations are conducted on a supercomputer using up to 2048 processors.

\section{Material and methods}

In the present study, we simulate a round liquid fuel jet into a chamber containing pressurized stationary gas. Two different fuels are considered, a standard European diesel and a biodiesel of soybean Methyl Ester. The fuel properties are given by Battistoni and Grimaldi [3]. Three different injection conditions, i.e., the jet radius and injection velocity, are investigated for each fuel. Therefore, in total six cases are simulated. The parameters are summarized in Table 1. The case names " $D$ " and "B" represent diesel and biodiesel fuels, respectively, where " $\mathrm{n}=1,2,3$ " indicates different injection conditions.

Table 1. Fuel properties and injection conditions of simulation cases.

\begin{tabular}{c|ccccc}
\hline Cases & $\begin{array}{c}\text { Fuel Density } \\
\rho_{l}\left(\mathrm{~kg} / \mathrm{m}^{3}\right)\end{array}$ & $\begin{array}{c}\text { Fuel Viscosity } \\
\mu_{l}(\mathrm{Pas})\end{array}$ & $\begin{array}{c}\text { Surface Tension } \\
\sigma(\mathrm{N} / \mathrm{m})\end{array}$ & $\begin{array}{c}\text { Jet Radius } \\
R_{\text {inj }}(\mathrm{m})\end{array}$ & $\begin{array}{c}\text { Injection Velocity } \\
U_{\text {inj }}(\mathrm{m} / \mathrm{s})\end{array}$ \\
\hline D1 & 825 & $2.10 \times 10^{-3}$ & 0.024 & $6.50 \times 10^{-5}$ & 70 \\
D2 & 825 & $2.10 \times 10^{-3}$ & 0.024 & $4.00 \times 10^{-5}$ & 50 \\
D3 & 825 & $2.10 \times 10^{-3}$ & 0.024 & $6.50 \times 10^{-5}$ & 50 \\
B1 & 870 & $3.90 \times 10^{-3}$ & 0.028 & $6.50 \times 10^{-5}$ & 70 \\
B2 & 870 & $3.90 \times 10^{-3}$ & 0.028 & $4.00 \times 10^{-5}$ & 50 \\
B3 & 870 & $3.90 \times 10^{-3}$ & 0.028 & $6.50 \times 10^{-5}$ & 50
\end{tabular}

The gas in the chamber is taken to be air under the pressure of $10^{6} \mathrm{~Pa}$ and the temperature $313.15 \mathrm{~K}$. The density and viscosity of the gas are $11.13 \mathrm{~kg} / \mathrm{m}^{3}$ and $1.46 \times 10^{5}(\mathrm{Pas})$, respectively. If the gas density, the jet radius, 
and the injection velocity are chosen to be reference scales, the key dimensionless parameters of problem can be obtained, which are shown in Table 2. The Reynolds and Weber numbers of the liquid jet are defined as $\operatorname{Re}_{l}=\rho_{l}\left(2 R_{i n j}\right) U_{i n j} / \mu_{l}$ and $\mathrm{We}_{l}=\rho_{l}\left(2 R_{i n j}\right) U_{i n j}^{2} / \sigma$, respectively, which measure the inertial effect compared to the viscous and surface tension forces. When $\mathrm{Re}_{l}$ and $\mathrm{We} l$ are large, the viscous and surface tension forces are insufficient to hold the injected fuel as a bulk liquid, and the liquid fuel will be atomized into small droplets. As the injected liquid will also induce flow motion of the gas in the chamber, the Reynolds number based on the gas properties, $\operatorname{Re}_{g}=\rho_{g}\left(2 R_{i n j}\right) U_{i n j} / \mu_{g}$, is to characterize the induced gas flow. When $\operatorname{Re}_{g}$ is large the gas flow can become turbulent. Finally, the liquid-to-gas density ratio is represented by $r$. Other dimensionless parameters can be computed based on these four parameters, for example, the liquid-to-gas viscosity ratio is equal to $r \operatorname{Re}_{g} / \operatorname{Re}_{l}$.

Table 2. Fuel properties and injection conditions of simulation cases.

\begin{tabular}{c|cccc}
\hline Cases & $\operatorname{Re}_{l}=\rho_{l}\left(2 R_{i n j}\right) U_{i n j} / \mu_{l}$ & $\mathrm{We}_{l}=\rho_{l}\left(2 R_{i n j}\right) U_{i n j}^{2} / \sigma$ & $r=\rho_{l} / \rho_{g}$ & $\operatorname{Re}_{g}=\rho_{g}\left(2 R_{i n j}\right) U_{i n j} / \mu_{g}$ \\
\hline D1 & $3.58 \times 10^{2}$ & $2.19 \times 10^{4}$ & 74.1 & $6.94 \times 10^{3}$ \\
D2 & $1.57 \times 10^{3}$ & $6.88 \times 10^{3}$ & 74.1 & $3.05 \times 10^{3}$ \\
D3 & $2.55 \times 10^{3}$ & $1.12 \times 10^{4}$ & 74.1 & $4.95 \times 10^{3}$ \\
B1 & $2.03 \times 10^{3}$ & $1.98 \times 10^{4}$ & 78.2 & $6.94 \times 10^{3}$ \\
B2 & $8.92 \times 10^{2}$ & $6.21 \times 10^{3}$ & 78.2 & $3.05 \times 10^{3}$ \\
B3 & $1.45 \times 10^{3}$ & $1.01 \times 10^{4}$ & 78.2 & $4.95 \times 10^{3}$
\end{tabular}

It can be observed from Tables 1 and 2 that the main difference between the diesel and biodiesel fuels lies at the fuel viscosity. The density and surface tension of the two fuels are quite similar. As a result, for a given injection condition, the diesel and biodiesel jets (such as $\mathrm{D} 1$ and $\mathrm{B} 1$ ) have similar $\mathrm{We}_{l}, \mathrm{Re}_{g}$, and $r$; while $\mathrm{Re}_{l}$ for the diesel is about two times of that for the biodiesel. It is expected a large liquid viscosity can damp small scale instability and thus weaken the atomization process [4]. It is the goal of the present study to investigate in detail the impact of the larger viscosity of the biodiesel to the atomization characteristics.

Thanks to the octree discretization of Basilisk, we can use a large domain for the present simulation so that the effects of boundaries to the atomizing jet are negligible. The computational domain for the atomizing jet is shown in Fig. 1. The fuel is injected into a cubic domain along the $z$ direction from the left boundary. A single mode perturbation is added to the inflow velocity as $U_{l, \text { inflow }}=U_{i n j}(1+\xi \sin (2 \pi t / \eta))$ to accelerate the development of the Kelvin-Helmholtz instability at the liquid-gas interface, where $\xi=0.05$ and $\eta=2.56 R_{i n j} / U_{i n j}$. The outflow boundary condition is invoked at the right boundary. All the rest boundaries of the domain are then taken as symmetric boundaries. The edge length of the domain is $96 R_{i n j}$. The minimum cell size of the adaptive mesh used is indicated by $\Delta_{\min }$. For cases D1, D2, B1, and B2, $\Delta_{\min }=R_{i n j} / 43$ and for cases D3 and B3, $\Delta_{\min }=R_{i n j} / 85$. A snapshot of the atomizing jet and the corresponding adaptive mesh are shown in Fig. 1. It can be seen that high mesh resolution is used to resolve the complex liquid-gas interface while the mesh away from the jet is coarsen to reduce computational costs. The total number of cells increases in time as more liquid is injected into the domain. The mesh shown in Fig. 1, which is a snapshot corresponding to $t U_{i n j} / R_{i n j}=49$, consist of about 50 million cells. It should be mentioned here that the liquid injection velocity used in the present study is lower than typical injection velocity in diesel engines. The usage of smaller injection velocity is simply to constrain the Reynolds number in the regime where a DNS is feasible with current computational resources. For practical injection velocity which can be 10 times of the present ones, the total number of cells would exceed 3 billion even with an adaptive mesh. Finally, the simulation data generated are visualized and analyzed by the open source package Vis/t [7].

\section{Results and discussion General behavior}

A snapshot of the atomizing liquid jet for the case 3 and $\Delta_{\min }=R_{i n j} / 85$ at $t U_{i n j} / R_{i n j}=47$ is shown in Fig. 2. When the liquid fuel is injected into the domain with a high speed, the impingement with the pressurized gas causes the liquid to spread laterally forming a liquid sheet. The liquid sheet moves backward so eventually the head of the liquid jet takes a mushroom shape, a closeup of which is given in Fig. 2. The liquid sheet of the mushroom head flaps and breaks violently. Ligaments are formed near the edge of the liquid sheet, which then in turn break into a large number of droplets. Different formation mechanisms of liquid ligaments have been discussed in previous works $[5,6]$. These mechanisms including the expansion of holes-in-liquid-sheets and the Rayleigh-Plateau instability of rims are also observed in the present simulation. The shapes of the liquid ligaments display strong variability and thus when they break the size of formed droplets is spread over a wide range. When the ligaments and droplets detach from the bulk liquid jet, their streamwise velocity decreases and they fall behind the mushroom head, forming a spray around the bulk liquid column.

Beyond the mushroom head, the interfacial waves on the bulk liquid column also produce droplets. Due to the shear between the moving liquid and the stagnant gas, a Kelvin-Helmholtz $(\mathrm{KH})$ instability develops. The $\mathrm{KH}$ interfacial waves are initially small and axisymmetric. As they are advected downstream, they grow in amplitude and roll backward like umbrellas. Lateral instability then show up and azimuthal variations are observed at the umbrella edge. When the waves propagate further downstream, holes forms in the liquid sheet and expansion of which finally causes the umbrella-shape waves break into ligaments, similar to the mushroom head but in a less violent manner. The orientations of the formed ligaments vary in a large extent. The ones more perpendicular to the jet 


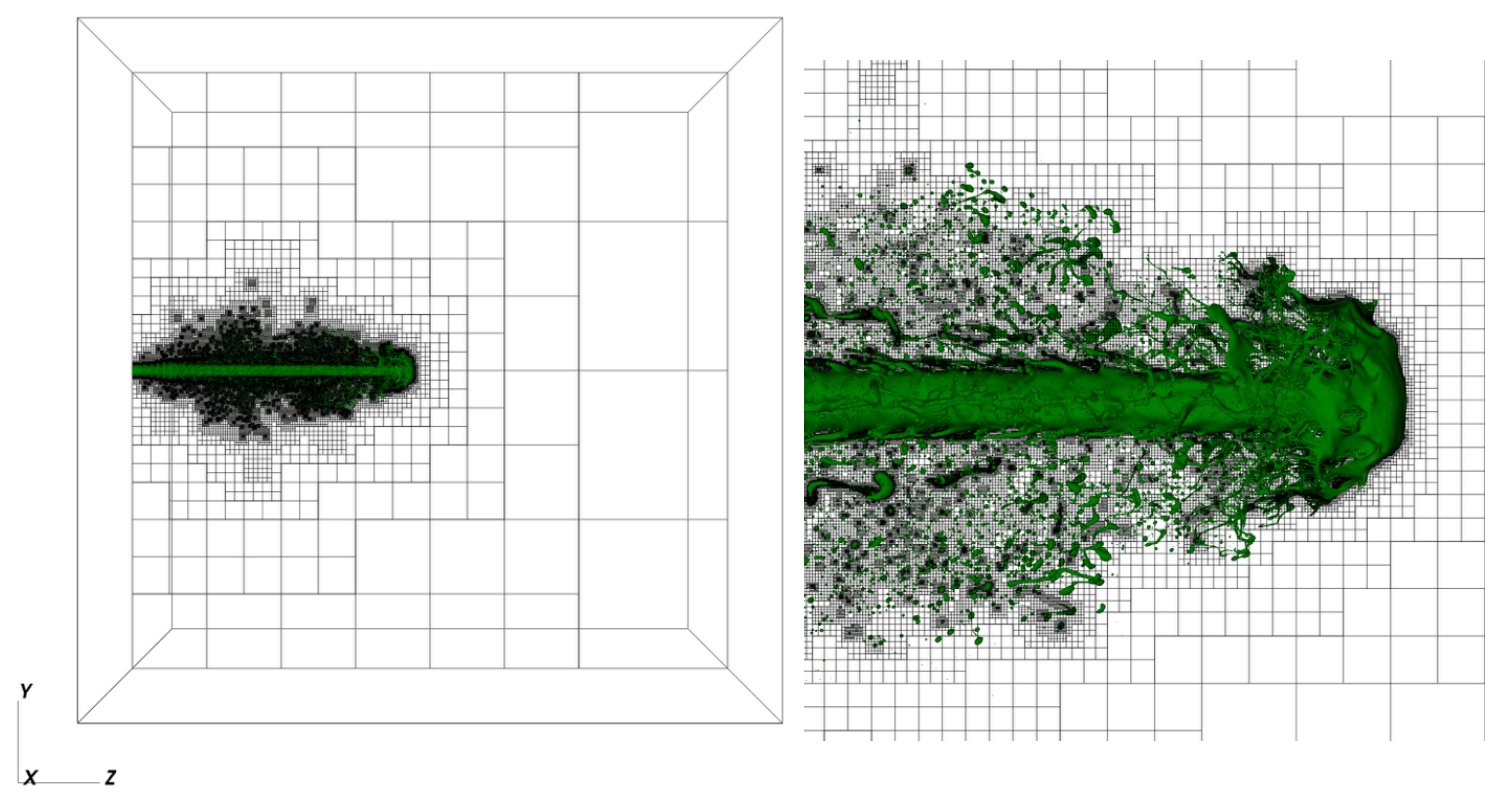

Figure 1. Simulation setup for an atomizing liquid jet and the adaptive mesh utilized.

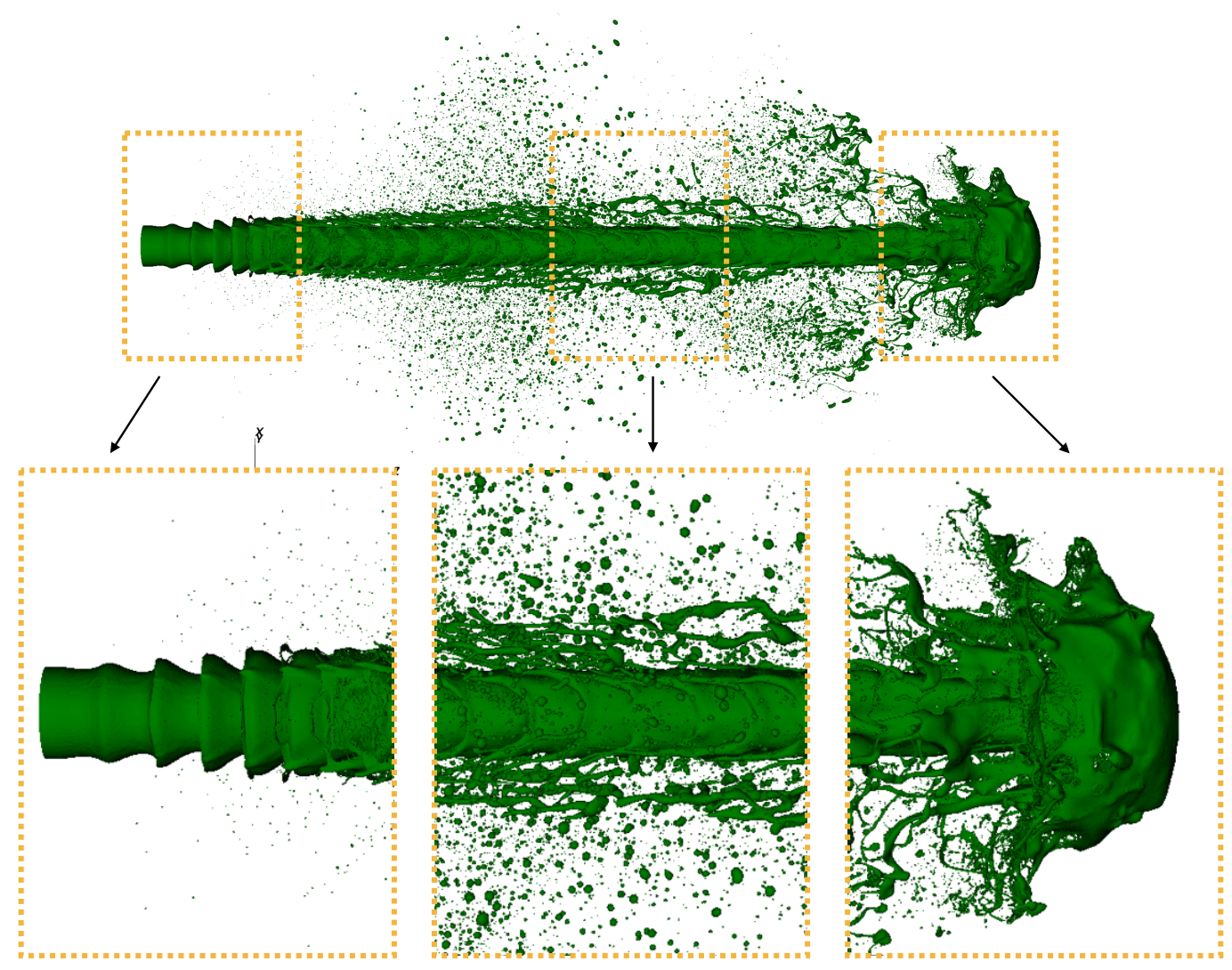

Figure 2. The atomizing jet for the case D3 at $t U_{i n j} / R_{i n j}=47$. The minimum cell size of the adaptive mesh is $\Delta_{\min }=R_{i n j} / 85$. 
breaks into droplets faster; while those more aligned to the jet tend to last for a longer time. Compared to the droplets formed at the mushroom head, the ones formed at the interfacial waves stay closer to the liquid jet and are less spread.

\section{Effect of grid resolution}

In order to verify whether the mesh resolution is sufficient to resolve the atomization process, the minimum cell size $\Delta_{\text {min }}$ is varied from $R_{i n j} / 21$ to $R_{i n j} / 85$ for cases D3 and B3. The results of the grid-refinement study are shown in Fig. 3. It can be observed that the results for the coarse mesh $\Delta_{\min }=R_{i n j} / 21$ are substantially different from the results for the other two meshes: the number of droplets produced is much lower and the ligaments formed at the interfacial waves are completely missed. The results for the intermediate and fine meshes $\Delta_{\min }=R_{\text {inj }} / 43$ and $R_{i n j} / 85$ are similar, although the ligaments formed at the edge of the mushroom head tends to break earlier for the intermediate mesh.

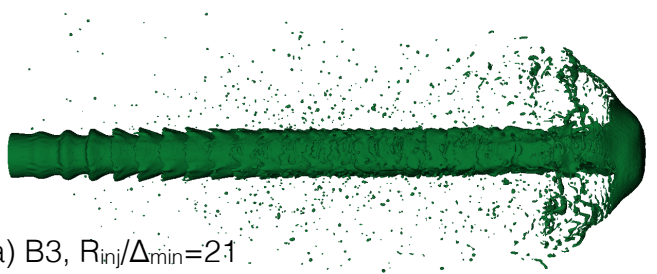

(a) B3, R Rinj $/ \Delta_{\min }=21$
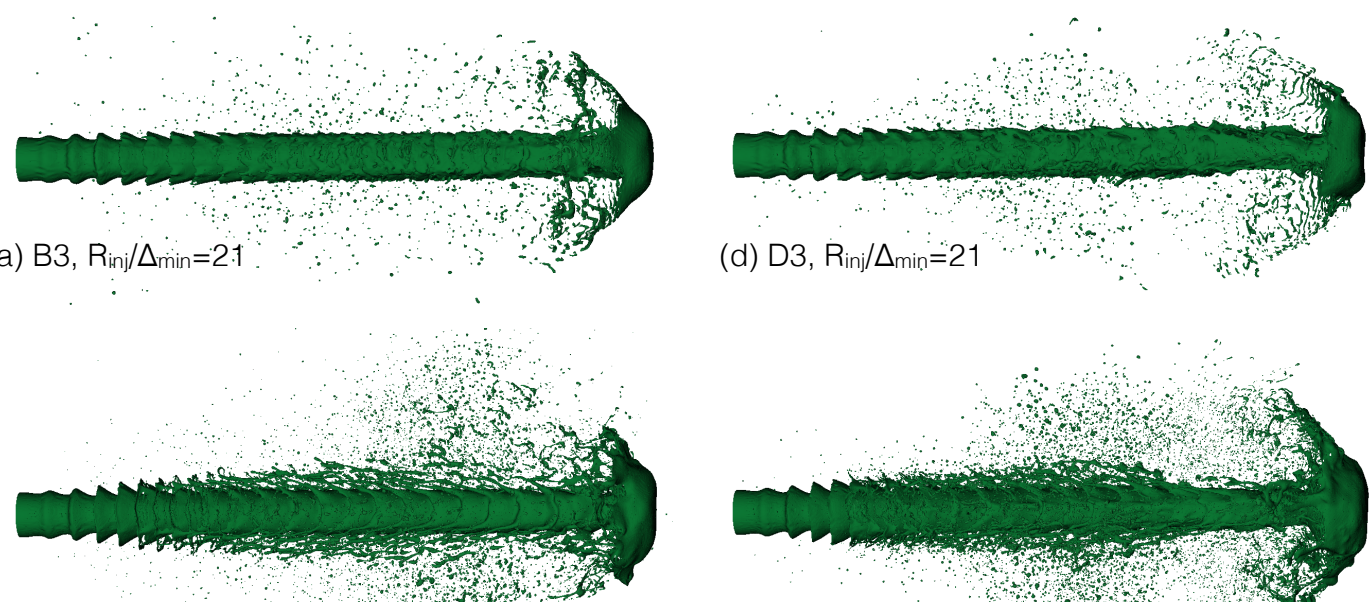

(b) $B 3, R_{\text {inj }} / \Delta_{\min }=43$
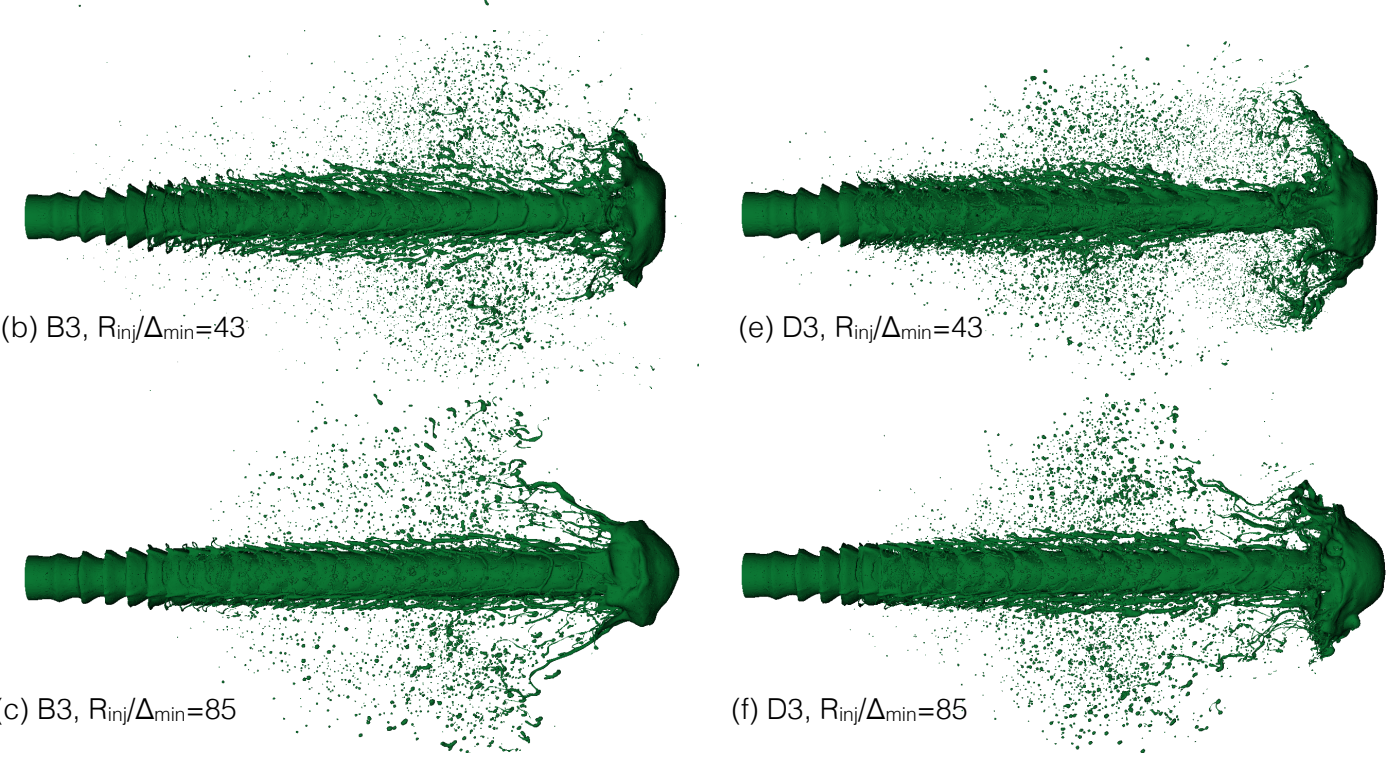

Figure 3. Snapshots of the atomizing biodiesel and diesel jets (B3 and D3) for different mesh resolutions: $R_{i n j} / \Delta_{\min }=21,43$, and 85 .

As $\operatorname{Re}_{g}$ is high for both cases B3 and D3, the gas flow around the liquid jet becomes turbulent. Figure 4 show the vortical structures by the $\lambda_{2}$ vortex-identification criterion proposed by Jeong and Hussain [8]. The turbulent vortical structures are most profound near the head of the liquid jet. When the mesh is refined, smaller vortical structures are captured. It is interesting to observe that actually less vortical structures are observed in the fine mesh results than in the coarse ones. As the cell size in simulations behaves like a cut-off scale that control the breakup of liquid sheets and ligaments. A coarse mesh will result in an earlier and more violent breakup, which thus will produce more vortical structures near the small liquid pieces. Up to the maximum simulation time in the present study, the liquid jet is in a transient process, toward being fully atomized. Therefore, the results here are insufficient to estimate the turbulence kinetic energy dissipation and the Kolmogorov length scale. Nevertheless as shown in Fig. 4, the simulation with the finest mesh is quite effective in capturing the small vortices generated.

\section{Effect of fuel viscosity to atomization}

From Fig. 3 it can also be observed that under the same injection condition, the biodiesel jet (B3) tends to produce thicker filaments and fewer droplets than the standard diesel (D3). The higher viscosity of the biodiesel is believed to be responsible for the discrepancy.

A more detailed comparison between the biodiesel and diesel atomizing jets is shown in Fig. 5. The two jets shown here correspond to the injection condition 3, i.e., $R_{i n j}=65 \mu \mathrm{m}$ and $U_{i n j}=50 \mathrm{~m} / \mathrm{s}$, see Tables 1 and 2 . While $\mathrm{We}_{l}, \mathrm{Re}_{g}$, and $r$ for the two cases are similar, $\operatorname{Re}_{l}=1450$ for the biodiesel is $43 \%$ lower than that for the diesel, I.e., $\operatorname{Re}_{l}=2550$. Several observations on the difference between the two cases can be made here. First, it is observed 
(a) $B 3, R_{\text {inj }} / \Delta_{\min }=21$

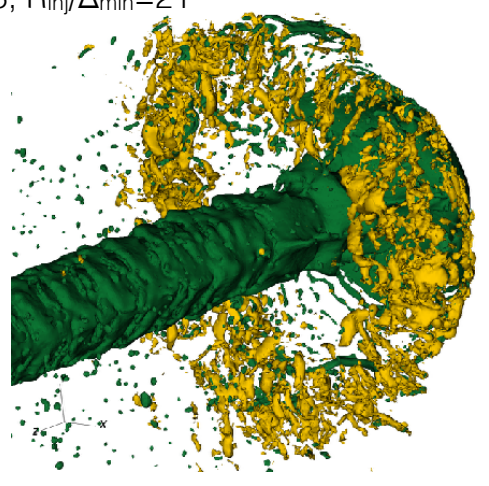

(b) B3, Rinj/ $\Delta_{\min }=43$

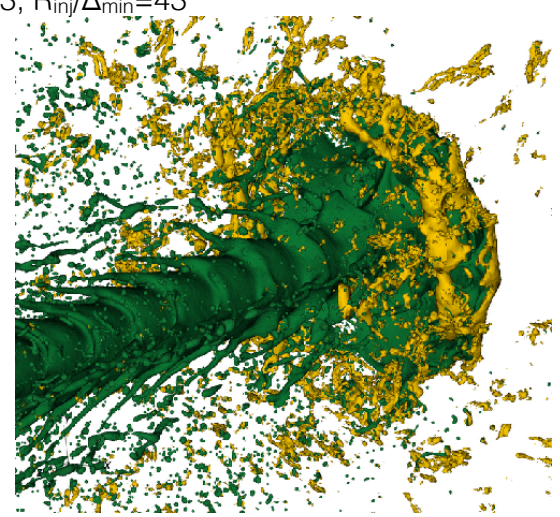

(c) $B 3, R_{\text {inj }} / \Delta_{\min }=85$

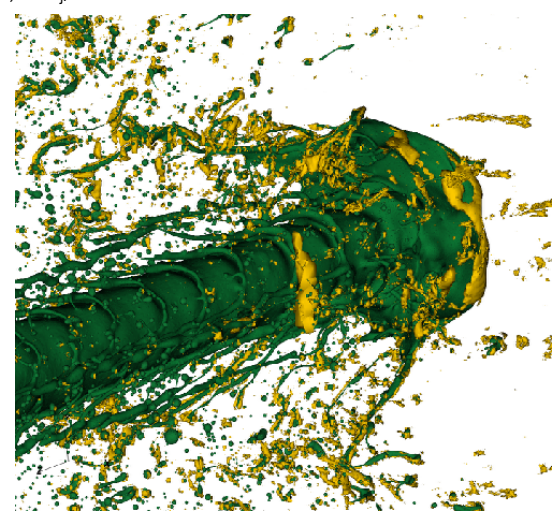

(d) $D 3, R_{\text {inj }} / \Delta_{\min }=21$

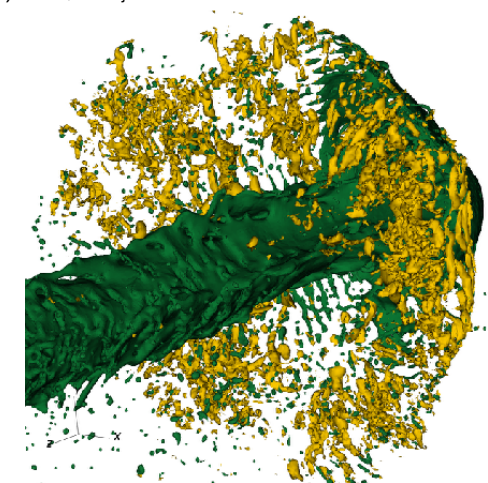

(e) D3, $R_{\text {in } j} / \Delta_{\min }=43$

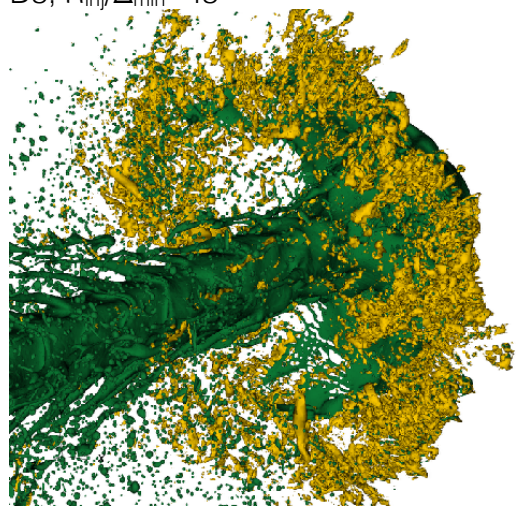

(f) $\mathrm{D} 3, \mathrm{R}_{\mathrm{in} j} / \Delta_{\min }=85$

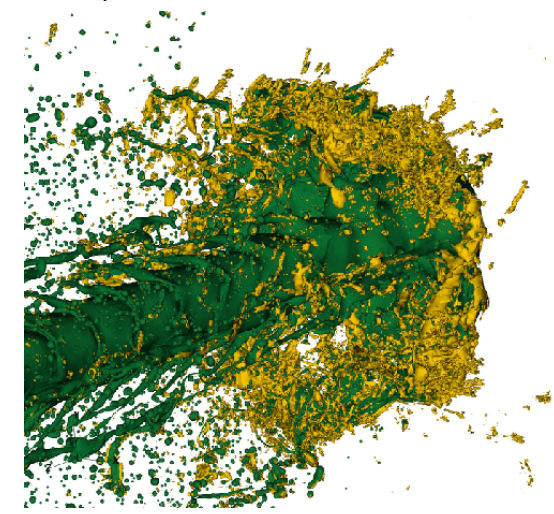

Figure 4. Snapshots of the vortical structures near heads of the biodiesel and diesel jets (B3 and D3) for different mesh resolutions: $R_{i n j} / \Delta_{\min }=21,43$, and 85 . 
that the holes in the interfacial waves on the bulk liquid column appear later for the biodiesel than the diesel. Holes are first observed on the fourth wave (counting from the left) for the diesel jet; while similar holes are not seen until the fifth wave for the biodiesel. Second, the ligaments formed in the biodiesel jet are thicker and longer for the biodiesel. The biodiesel ligaments also seem to last for longer before they break into droplets, see the second row of Fig. 5, indicating that the higher viscosity of the biodiesel is effective in damping small scale instability and delaying ligament breakup. Third, the lateral radius of the mushroom head is smaller for the biodiesel and the roll-up sheets sustain longer and less holes are observed. Compared to the diesel analog, much more ligaments attaching to the edge of the mushroom head are observed for the biodiesel. These ligaments are also longer and thicker. As a result of the delay of sheet and ligament breakups, much less droplets are formed behind the mushroom head for the biodiesel than the diesel.
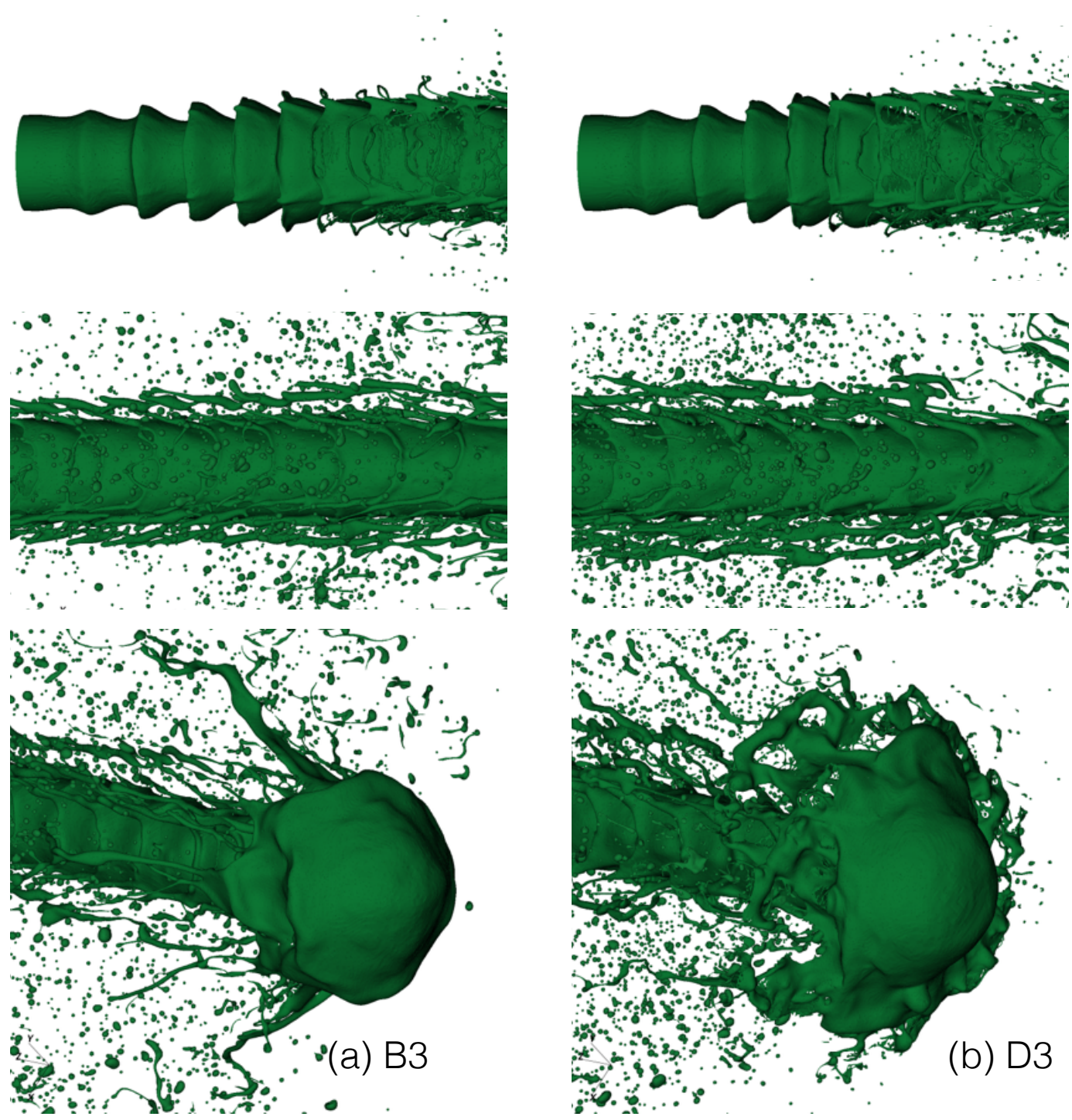

Figure 5. Comparison of biodiesel and diesel jets under identical injection conditions $\left(R_{i n j}=65 \mu \mathrm{m}\right.$ and $\left.U_{i n j}=50 \mathrm{~m} / \mathrm{s}\right)$.

\section{Droplet size distribution}

The statistics of the droplets formed are also investigated since which is an important to many atomization applications. Figure 6 shows the size distribution of droplet number $N_{p}$ for cases B3 and D3 at $t U_{i n j} / R_{i n j}=47$. The droplet diameter is calculated as $d_{v}=\left(6 V_{p} / \pi\right)^{1 / 3}$, where $V_{p}$ is the droplet volume and which is in turn computed by tagging the volume-fraction function and identifying the connected liquid structures. The histogram plots shown in Figs. 6(a)-(b) are for B3 and different mesh resolutions varying from $R_{i n j} / \Delta_{\min }=21$ to 85 . The bin width is 0.75 $\mu \mathrm{m}$, which is about the minimum cell size of the fine mesh. It is shown that more small droplets are produced when the mesh is refined. However, as the droplets with diameter similar to the cell size are affected by the numerical error (such as fake breakup due to lack of mesh resolution) and thus the accuracy of results for these small droplets are less trustworthy. If these small droplets $\left(d_{v}<3 \mu \mathrm{m}\right)$ are ignored, then it is found that the intermediate and fine meshes actually yield similar results, see Figs. $6(d)$ and $(e)$, which means that we have obtained converged results 
for the droplet size distribution with $R_{i n j} / \Delta_{\min }=85$.

For $d_{v}>3 \mu \mathrm{m}, N_{p}$ decreases with $d_{v}$ and the profile matches well with the exponential function $\exp \left(-d_{v} / \lambda\right)$ for both cases B3 and D3, where $\lambda$ is a length scale characterizing the shape of PDF. When $\lambda$ decreases, the decay of $N_{d}$ with increasing $d_{v}$ is faster, indicating the droplet size distribution inclines more toward smaller droplets, or in other words the liquid jet is "more seriously atomized". It is fitted by the data for the finest mesh that, $\lambda$ is about 2.7 $\mu \mathrm{m}$ for the biodiesel and about $2.2 \mu \mathrm{m}$ for the diesel, see Fig. 6(f). The larger $\lambda$ for the biodiesel is consistent with the previous observation of the less "intense" atomization of biodiesel due to its higher viscosity.

Under the same injection conditions, significantly fewer smaller droplets are formed in the biodiesel jet. Note that $N_{d}$ in Fig. 6 is plotted in the logarithmic scale, the difference of droplet number between the two fuels may look small, but is indeed very large. As an example, for droplet diameter between 4.5 and $5.25 \mu \mathrm{m}, N_{p}=517$ for B3 and $N_{p}=897$ for D3. Namely, the biodiesel jet produces $42 \%$ less droplets for this size range $4.5-5.25 \mu \mathrm{m}$ compared to the standard diesel fuel.
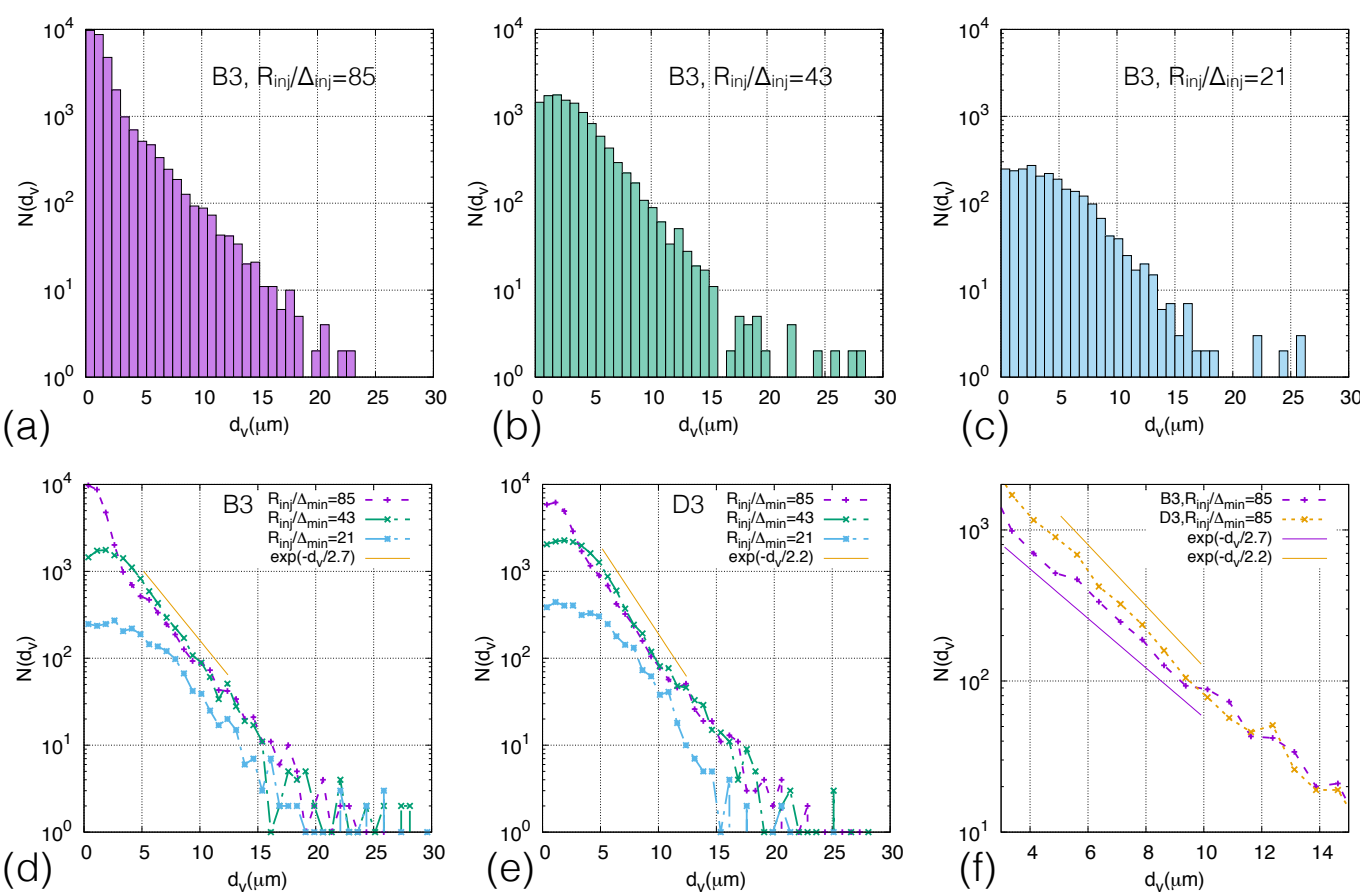

Figure 6. Droplet size distribution for B3 and D3 at $t U_{i n j} / R_{i n j}=47$. (a)-(c) are for B3 with different $\Delta_{\min }$.

\section{Effect of injection conditions}

Finally, we examine the effect of the fuel viscosity on atomization for different injection conditions. Compared to $\mathrm{B} 3 / \mathrm{D} 3$, the injection velocity for B1/D1 is higher and as a result $\mathrm{Re}_{l}$ and $\mathrm{We}_{l}$ are larger, see Table 2. In contrast, the jet radius $R_{i n j}$ for $\mathrm{B} 2 / \mathrm{D} 2$ is smaller than $\mathrm{B} 3 / \mathrm{D} 3$, resulting smaller $\mathrm{Re}_{l}$ and $\mathrm{We}_{l}$. The size distribution of droplet number for these three injection conditions are shown in Fig. 7. The mesh resolution is the same for all cases shown here, i.e., $\Delta_{\min }=R_{\text {inj }} / 43$. When $\mathrm{Re}_{l}$ and $\mathrm{We}_{l}$ decrease in the order of B1/D1, B3/D3, and B2/D2, the number of droplets generated decreases accordingly. The discrepancy of $N_{p}$ between the biodiesel and diesel jets becomes more profound when $\mathrm{Re}_{l}$ and $\mathrm{We}_{l}$ decrease. For $d_{p}>10 \mu \mathrm{m}$, the difference of $N_{p}$ between D1 and B1 is barely seen. In contrast, the difference between D2 and B2 is much more profound. This seems to indicate that for sufficiently large $\mathrm{Re}_{l}$ and $\mathrm{We}_{l}$, the liquid jet is atomized violently and the liquid viscosity has little effect on the size distribution of formed droplets. For that case, the difference of the droplet statistics between the diesel and the biodiesel is small. On the other hand, when $\mathrm{Re}_{l}$ and $\mathrm{We}_{l}$ are small, the atomization of the liquid jet and the resulting droplet statistics are more sensitive to the liquid viscosity. Then replacing the standard diesel with the biodiesel in fuel injection can introduce a significant impact to the droplet size distribution.

\section{Conclusions}

Three dimensional direct numerical simulations are performed in the present work to investigate the effect of fuel properties on the atomization characteristics. Atomizing liquid jets for both the standard diesel and the biodiesel under different injection conditions are considered. Grid-dependent study is conducted, showing that the present mesh resolution is sufficient to capture droplets larger than $6 \mu \mathrm{m}$. Generally, a lower number of droplets are formed in a biodiesel jet compared to those in the diesel jet under the same injection condition due to the higher viscosity of the biodiesel. The size distribution of droplet number is also presented. The decay of droplet number with droplet diameter follows the exponential function $\exp \left(-d_{v} / \lambda\right)$ for both the biodiesel and diesel fuels. The $\lambda$ is a length scale that characterizes the shape of distribution function and $\lambda$ for the biodiesel is larger than that for the diesel under the 
(a)

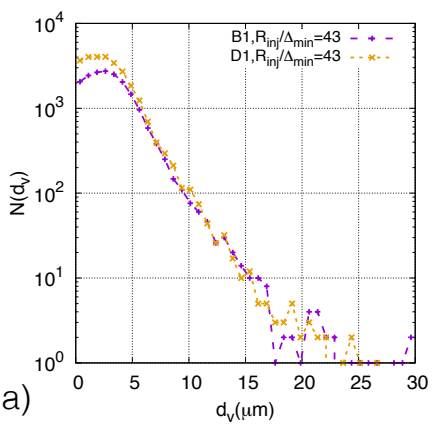

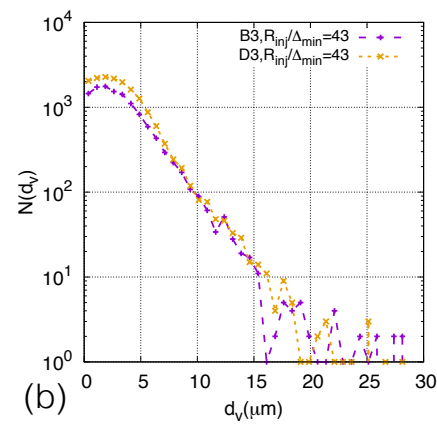

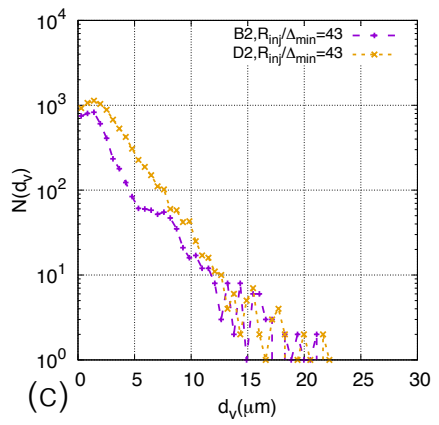

Figure 7. Droplet size distribution for B1-3 and D1-3 at $t U_{i n j} / R_{i n j}=47$ for $\Delta_{\min }=R_{i n j} / 43$.

same injection condition. Finally, the effect of injection condition is also studied, showing that the difference of the droplet statistics between the biodiesel and the diesel decreases with the liquid jet Reynolds and Weber numbers.

\section{Acknowledgements}

This project has been supported by the ANR MODEMI project (ANR-11-MONU-0011) program. This work was granted access to the HPC resources of TGCC-CURIE under the allocations x20152b7325, x20162b7325 and t20162b7760 made by GENCI. We would also acknowledge support from the Academic and Research Computing Services at the Baylor University.

\section{References}

[1] Popinet S., The Basilisk code, Available from http://basilisk.fr/.

[2] Popinet, S., 2009, J. Comput. Phys., 228, pp. 5838-5866.

[3] Battistoni, M. and Grimaldi, C. N., 2012, Appl. Energy, 97, pp. 656-666.

[4] Müller, T., Sänger, A., Habisreuther, P., Jakobs, T., Trimis, D., Kolb, T. and Zarzalis, N., 2016, Int. J. Multiphase Flow, 87, pp. 212-228.

[5] Shinjo, J. and Umemura, A., 2010, Int. J. Multiphase Flow, 36, pp. 513-532.

[6] Ling, Y. and Fuster, D. and Zaleski, S. and Tryggvasson, G., 2017, Phys. Rev. Fluids, 2, pp. 014005.

[7] Childs H., Brugger, E., Whitlock, B., Meredith J., Ahern S., Pugmire D., Biagas K., Miller, M., Harrison, C., Weber, G. H., Krishnan, Hari, Fogal, T. , Sanderson, A. , Garth, C., Bethel, E. W., Camp, D. , Rübel, O., Durant,M., Favre, J. M. and Navrátil P., 2012, Vislt: An End-User Tool For Visualizing and Analyzing Very Large Data, High Performance Visualization-Enabling Extreme-Scale Scientific Insight, pp. 357-372.

[8] Jeong, J. and Hussain, F., 1995, J. Fluid Mech., 285, pp. 69-94. 